\title{
COMBINATORIAL RICCI FLOWS WITH APPLICATIONS TO THE HYPERBOLIZATION OF CUSPED 3-MANIFOLDS
}

\author{
KE FENG, HUABIN GE, AND BOBO HUA
}

\begin{abstract}
In this paper, we adopt combinatorial Ricci curvature flow methods to study the existence of cusped hyperbolic structure on 3-manifolds with torus boundary. For general pseudo 3-manifolds, we prove the long-time existence and the uniqueness for the extended Ricci flow for decorated hyperbolic polyhedral metrics. We prove that the extended Ricci flow converges to a decorated hyperbolic polyhedral metric if and only if there exists a decorated hyperbolic polyhedral metric of zero Ricci curvature. If it is the case, the flow converges exponentially fast. These results apply for cusped hyperbolic structure on 3-manifolds via ideal triangulation.
\end{abstract}

\section{INTRODUCTION}

We continue our program to study the hyperbolization of 3-manifolds using combinatorial Ricci flow methods. In the earlier paper [FGH20], we obtained a unique complete hyperbolic structure and a geometric triangulation on a compact 3-manifolds whose boundary components have genus at least 2 , under the assumption that there is a triangulation with edge valences at least 10. In this paper, we consider cusped hyperbolic 3-manifolds, which are noncompact complete hyperbolic 3-manifolds with cusp ends and finite volume. Given a compact 3-manifold $N$ with torus boundary components, we consider the problem whether there is a cusped hyperbolic structure on $N-\partial N$. Given an ideal triangulation for $N$, we show that the following are equivalent: the existence of a cusped hyperbolic structure compatible with the triangulation and the convergence of the combinatorial Ricci flow.

In the seminar work [Thu79], Thurston introduced 2-dimensional circle packings to construct hyperbolic manifolds or orbifolds. Chow and Luo [CL03] introduced a combinatorial Ricci flow for triangulated surfaces. For a compact triangulated surface of non-positive Euler characteristic, they proved that the Ricci flow with any initial circle packing metric exists for all times and converges exponentially fast to Koebe-Andreev-Thurston's circle packing metrics. To extend Thurston's circle packings to higher dimensional cases, Cooper and Rivin [CR96] studied ball packings of 3-manifolds. Inspired by the work of Chow and Luo, Glickenstein [Gli05a, Gli05b] introduced a combinatorial Yamabe flow based on Euclidean triangulations defined by ball packings. The second author, Jiang and Shen [GJS18] studied the convergence of Glickenstein's Yamabe flow for regular ball packings in Euclidean background geometry. The second and third authors [GH20] studied the combinatorial Yamabe flow for triangulations of closed 3-manifolds 
in hyperbolic background geometry and proved the convergence of the flow under some combinatorial assumption.

We recall the setting of 3-dimensional triangulated spaces. Let $\left\{T_{1}, \cdots, T_{t}\right\}, t \in \mathbb{N}$, be a finite collection of combinatorial tetrahedra and $\mathscr{T}$ be the disjoint union $T_{1} \sqcup \cdots \sqcup T_{t}$, which is a simplicial complex. The quotient space $(M, \mathcal{T})=\mathscr{T} / \sim$, via a family of affine isomorphisms pairing faces of tetrahedra in $\mathscr{T}$, is called a compact pseudo 3-manifold $M$ together with a triangulation $\mathcal{T}$. Note that $\mathcal{T}$ consists of equivalent classes of simplexes in $\mathscr{T}$. Pseudo 3-manifolds are very general concepts, which include manifolds with triangulation as special cases. $M$ is called a closed pseudo 3-manifold if each codimension-1 face of tetrahedra in $\mathscr{T}$ is identified with another codimension-1 face. We denote by $V=V(\mathcal{T})$ (resp. $E=E(\mathcal{T})$ ) the set of vertices (resp. edges) in $\mathcal{T}$. We define the valence of an edge $e \in E$, denoted by $d_{e}$, to be the number of edges in $\mathscr{T}$ in the equivalent class of $e$.

An ideal tetrahedron in $\mathbb{H}^{3}$ is a convex hull of 4 points $\left\{v_{1}, v_{2}, v_{3}, v_{4}\right\}$ on $\partial \mathbb{H}^{3}$ in generic position. A decorated ideal tetrahedron defined by Penner [Pen87] is a pair $\left(s,\left\{H_{1}, H_{2}, H_{3}, H_{4}\right\}\right)$ where $s$ is an ideal tetrahedron and $H_{i}$ is an 2-horosphere centered at the vertex $v_{i}$ for any $1 \leq i \leq 4$. For each edge $e=v_{i} v_{j}$ in a decorated ideal tetrahedron we assign the signed length $l_{i j}$ as follows: the absolute value $\left|l_{i j}\right|$ of the length is the distance between $H_{i} \cap e$ and $H_{j} \cap e, l_{i j}>0$ if $H_{i}$ and $H_{j}$ are disjoint, and $l_{i j} \leq 0$ if $H_{i} \cap H_{j} \neq \emptyset$. Codimension- 1 faces of decorated ideal tetrahedron are decorated ideal triangles in totally geodesic hyperbolic planes.

Definition 1.1. A decorated hyperbolic polyhedral metric, decorated metric in short, on a closed pseudo 3-manifold $(M, \mathcal{T})$ is obtained by replacing each tetrahedra in $\mathcal{T}$ by a decorated ideal tetrahedron and replacing the affine gluing homeomorphisms by isometries preserving the decoration, i.e. gluing decorated ideal tetrahedra along codimension-1 faces. We denote by $l=$ $\left(l\left(e_{1}\right), \ldots, l\left(e_{m}\right)\right)$, where $E=\left\{e_{1}, \ldots, e_{m}\right\}$, the (signed) edge length vector of a decorated metric, and by $\mathscr{L}(M, \mathcal{T}) \subset \mathbb{R}^{E}$ the set of all decorated metrics on $(M, \mathcal{T})$ parametrized by the edge length vector $l$. The Ricci curvature $K_{e}(l)$ of the metric l assigned to each edge e is $2 \pi$ minus the cone angle at the edge e, i.e. the total dihedral angle surrounding the edge e. We write $K(l)=\left(K_{e_{1}}(l), \ldots, K_{e_{m}}(l)\right)$.

The main purpose is to find decorated metrics $l$ with no singularity on edges, i.e. $K_{e}(l)=0$ for all $e \in E$, called zero-curvature decorated metrics. We recall the motivation for the above construction in the literature, see e.g. [Luo05, LY18]. Suppose that $N$ is a compact 3-manifold with boundary. The purpose is to find a hyperbolic metric of finite volume on $N-\partial N$ with cusp ends. Let $C(N)$ be the compact 3-space obtained by coning off each boundary component of $N$ to a point. In particular, if $N$ has $k$ boundary components, then there are exactly $k$ cone points $\left\{v_{1}, \ldots, v_{k}\right\}$ in $C(N)$ so that $C(N)-\left\{v_{1}, \ldots, v_{k}\right\}$ is homeomorphic to $N-\partial N$. An ideal triangulation $\mathcal{T}$ of $N$ is a triangulation $\mathcal{T}$ of $C(N)$ such that the vertices of the triangulation are exactly the cone points $\left\{v_{1}, \ldots, v_{k}\right\}$. By Moise [Moi52], every such compact 3-manifold $N$ can be ideally triangulated. In our terminology, $(C(N), \mathcal{T})$ is a closed pseudo 3-manifold and 
$N$ is homeomorphic to $C(N)-s t\left(v_{1}, \ldots, v_{k}\right)$, where $s t\left(v_{1}, \ldots, v_{k}\right)$ is the open star of the vertices $\left\{v_{1}, \ldots, v_{k}\right\}$ in the second barycentric subdivision of the triangulation $\mathcal{T}$. As in Definition 1.1, we can endow $(C(N), \mathcal{T})$ with various decorated metrics. If there is a decorated metric $l \in$ $\mathscr{L}(C(N), \mathcal{T})$ with zero Ricci curvature at edges, then we obtain a hyperbolic metric on $N-\partial N$ with cusp ends via gluing these ideal tetrahedra. We call this a cusped hyperbolic structure on $N$ associated with the ideal triangulation $\mathcal{T}$.

Motivated by Chow and Luo [CL03] and Luo [Luo05], for a compact 3-manifold with boundary equipped with ideal triangulation, or more generally a closed pseudo 3-manifolds $(M, \mathcal{T})$, we consider the following combinatorial Ricci flow $l(t) \in \mathscr{L}(M, \mathcal{T})$ to study the existence of hyperbolic metrics,

$$
\frac{d}{d t} l(t)=K(l(t)), \quad \forall t \geq 0
$$

This flow was first proposed by Luo [Luo05] in the hyper-ideal setting and further studied in the decorated setting by [Yan19]. This is a negative gradient flow of a local convex function on $\mathscr{L}(M, \mathcal{T})$, related to the so-called co-volume function. The difficulty is that the domain $\mathscr{L}(M, \mathcal{T})$ is not convex in $\mathbb{R}^{E}$.

To circumvent the difficulty, Luo and Yang [LY18] extended the set of decorated metrics to a general framework. A generalized decorated tetrahedron is a (topological) tetrahedron of vertices $\left\{v_{1}, \ldots, v_{4}\right\}$ so that each edge $v_{i} v_{j}$ is assigned a real number $l_{i j}=l_{j i}, 1 \leq i \neq j \leq 4$, called the (signed) length. The space of all generalized decorated tetrahedra parameterized by the edge length vectors $l=\left(l_{12}, \ldots, l_{34}\right)$ is $\mathbb{R}^{6}$. For a generalized decorated tetrahedron, Luo and Yang [LY18] defined the extended dihedral angles, still denoted by $\alpha_{i j}: \mathbb{R}^{6} \rightarrow \mathbb{R}$, extending dihedral angles for decorated ideal tetrahedra, which turns out to be continuous functions of the edge lengths $l_{i j}$; see Section 2 .

Definition 1.2. Let $(M, \mathcal{T})$ be a closed pseudo 3-manifold. We call any $l \in \mathbb{R}^{E}$ a generalized decorated hyperbolic polyhedral metric, generalized decorated metric in short, on $(M, \mathcal{T})$, which replaces each tetrahedron in $\mathcal{T}$ by a generalized decorated ideal tetrahedron with (signed) edge lengths given by $l$. The generalized Ricci curvature of an edge e, denoted by $\widetilde{K}_{e}(l)$, is defined similarly as in Definition 1.1 by using extended dihedral angles on edges, and we write the generalized Ricci curvature vector as $\widetilde{K}(l)$.

We say that $l \in \mathbb{R}^{E}$ is a zero-curvature generalized decorated metric if $\widetilde{K}(l)=0$. The extended Ricciflow $l(t) \in \mathbb{R}^{E}$ is defined as follows,

$$
\frac{d}{d t} l(t)=\widetilde{K}(l(t)), \quad \forall t \geq 0
$$

We prove the long-time existence and the uniqueness of the extended Ricci flow. 
Theorem 1.3. For a closed pseudo 3-manifold $(M, \mathcal{T})$ and any initial generalized decorated metric $l_{0} \in \mathbb{R}^{E}$, there exists a unique solution $\{l(t) \mid t \in[0, \infty)\} \subset \mathbb{R}^{E}$ to the extended Ricci flow (1.2).

The following are main results of the paper.

Theorem 1.4. For a closed pseudo 3-manifold $(M, \mathcal{T})$, let $\{l(t)\}_{t \geq 0}$ be a solution to the extended Ricciflow (1.2).

(1) If there is no zero-curvature generalized decorated metrics, then $l(t)$ diverges to infinity in subsequence, i.e. there exists a subsequence $t_{n} \rightarrow \infty$ such that $\left|l\left(t_{n}\right)\right| \rightarrow \infty, n \rightarrow \infty$.

(2) There is a zero-curvature decorated metric if and only if $l(t)$ converges to a zero-curvature decorated metric. If it is the case, the convergence is exponentially fast.

Remark 1.5. (i) The first assertion gives a necessary condition for the non-existence result of zero-curvature generalized decorated metrics.

(ii) By the second assertion, the extended Ricci flow provides an effective method for numerically computing zero-curvature decorated metrics.

Note that a finite cusped 3-manifold with an ideal triangulation is a closed pseudo 3-manifold. The following theorem is a consequence of Theorem 1.4

Theorem 1.6. For a finite cusped 3-manifold $N$ with an ideal triangulation $\mathcal{T}$, let $\{l(t)\}_{t \geq 0}$ be a solution to the extended Ricci flow (1.2).

(1) If there is no cusped hyperbolic structure on $N$ associated with $\mathcal{T}$, then $l(t)$ diverges to infinity in subsequence, i.e. there exists a subsequence $t_{n} \rightarrow \infty$ such that $\left|l\left(t_{n}\right)\right| \rightarrow$ $\infty, n \rightarrow \infty$.

(2) There is a cusped hyperbolic structure on $N$ so that $\mathcal{T}$ is isotopic to a geometric triangulation if and only if $l(t)$ converges to a decorated metric. Moreover, the flow converges exponentially fast in this case, and the cusped hyperbolic structure on $N$ is unique by the famous Mostow-Prasad rigidity theorem.

The key ingredient of the proof is that the extended Ricci flow is the negative gradient flow of a convex function on $\mathbb{R}^{E}$, related to the co-volume function. The function appeared before in Cohn, Kenyon, and Propp [CKP01], and Bobenko, Pinkahl and Springborn [BPS15], and Luo and Yang [LY18].

A closed pseudo 3-manifold $(M, \mathcal{T})$ is called edge-transitive if for any edges $e, \hat{e} \in E$, there exists an automorphism of the triangulation $\mathcal{T}$ which maps $e$ to $\hat{e}$. This yields that $d_{e}=d_{\hat{e}}$ for any $e, \hat{e} \in E$. We obtain the following corollary of Theorem 1.4

Corollary 1.7. Let $(M, \mathcal{T})$ be an edge-transitive closed pseudo 3-manifold, and $d=d_{e}$ for some $e \in E$.

(1) If $d \neq 6$, then there exists no zero-curvature generalized decorated metrics, and the extended Ricci flow $l(t)$ diverges to infinity in subsequence. 
(2) If $d=6$, then there exists a zero-curvature decorated metric, and the extended Ricci flow $l(t)$ converges to a zero-curvature decorated metric exponentially fast.

The paper is organized as follows. In Section 2, we collect basic facts on the geometry of decorated ideal tetrahedra, including volume and co-volume functions, etc. In Section 3, we study the extended Ricci flow and prove the main results of the paper.

Acknowledgements We thank Yi Liu, Feng Luo, Jiming Ma, Tian Yang for many discussions on related problems in this paper.

H. G. is supported by NSFC, no. 11871094. B. H. is supported by NSFC, no.11831004 and no. 11926313 . F. K. is supported by NSFC, no. 11901009.

\section{PRELIMINARIES}

We recall some results on decorated ideal tetrahedra; see Luo and Yang [LY18]. An ideal tetrahedron in $\mathbb{H}^{3}$ is determined up to isometry by its six dihedral angles on edges. These angles satisfy the condition that angles at opposite edges are the same and the sum of all angles is $2 \pi$. Thus the set of all ideal tetrahedra modulo isometry can be identified with $\{(a, b, c) \in$ $\left.\mathbb{R}_{>0}^{3} \mid a+b+c=\pi\right\}$.

Let $\left(s,\left\{H_{1}, H_{2}, H_{3}, H_{4}\right\}\right)$ be a decorated ideal tetrahedron with signed edge length $l_{i j}=l_{j i}$, where $s$ is an ideal tetrahedron $\left\{v_{1}, v_{2}, v_{3}, v_{4}\right\}$ and $H_{i}, 1 \leq i \leq 4$, are 2-horospheres centered at $v_{i}$. Note that for any $i, s \cap H_{i}$ is isometric to a Euclidean triangle.

The following result is well-known; see e.g. [Luo11a, Lemma 2.5] or [BPS15, Lemma 4.2.3]

Lemma 2.1. Suppose that $l_{i j}$ are the edge lengths of a decorated ideal tetrahedron $\sigma=\left(s,\left\{H_{1}, H_{2}, H_{3}, H_{4}\right\}\right)$. Then all four Euclidean triangles $\left\{s \cap H_{i}\right\}$ are similar to the Euclidean triangle $\tau$ of edge lengths $e^{\left(l_{i j}+l_{k h}\right) / 2}, e^{\left(l_{i k}+l_{j h}\right) / 2}$, and $e^{\left(l_{i h}+l_{j k}\right) / 2}$, so that the triangle inequalities hold, i.e.

$$
e^{\left(l_{i j}+l_{k h}\right) / 2}+e^{\left(l_{i k}+l_{j h}\right) / 2}>e^{\left(l_{i h}+l_{j k}\right) / 2},
$$

for $\{i, j, k, h\}=\{1,2,3,4\}$. The dihedral angle $\alpha_{i j}$ of $\sigma$ at the edge $v_{i} v_{j}$ is equal to the inner angle of $\tau$ opposite to the edge of length $e^{\left(l_{i j}+l_{k h}\right) / 2}$. Conversely, if $\left(l_{12}, \ldots, l_{34}\right) \in \mathbb{R}^{6}$ satisfies the above triangle inequalities, then there is a unique decorated ideal tetrahedron having $l_{i j}$ as the lengths.

The set of all decorated ideal tetrahedra can be parametrized by the edge length as follows,

$$
\mathscr{L}:=\left\{\left(l_{12}, \cdots, l_{34}\right) \in \mathbb{R}^{6}: e^{\left(l_{i j}+l_{k h}\right) / 2}+e^{\left(l_{i k}+l_{j h}\right) / 2}>e^{\left(l_{i h}+l_{j k}\right) / 2},\{i, j, k, h\} \text { distinct }\right\} .
$$

Luo and Yang [LY18] extended it to the set of generalized decorated ideal tetrahedra $\left\{\left(l_{12}, \cdots, l_{34}\right) \in\right.$ $\left.\mathbb{R}^{6}\right\}$. A generalized decorated ideal tetrahedron is characterized by the length vector $\left(l_{12}, \cdots, l_{34}\right) \in$ $\mathbb{R}^{6}$. To define dihedral angles and the volume of a generalized decorated tetrahedron, we need to use the notion of generalized Euclidean triangles and their angles. A generalized Euclidean 
triangle is a (topological) triangle of vertices $\left\{v_{1}, v_{2}, v_{3}\right\}$ so that each edge is assigned a positive number; let $x_{i}$ be the assigned length of the edge $v_{j} v_{k}$ where $\{i, j, k\}=\{1,2,3\}$. The inner angle $a_{i}=a_{i}\left(x_{1}, x_{2}, x_{3}\right)$ at the vertex $v_{i}$ is the inner angle of the Euclidean triangle of edge lengths $x_{1}, x_{2}, x_{3}$ opposite to the edge of length $x_{i}$, if the triangle inequalities hold; or $a_{i}=\pi, a_{j}=a_{k}=0$, if $x_{i} \geq x_{j}+x_{k}$.

Lemma 2.2. (Luo [Luo11b] $)$ The angle function $a_{i}\left(x_{i}, x_{j}, x_{k}\right): \mathbb{R}_{>0}^{3} \rightarrow[0, \pi]$ is continuous so that $a_{1}+a_{2}+a_{3}=\pi$ and the $C^{0}$ - smooth differential 1-form $\sum_{i=1}^{3} a_{i} d\left(\ln x_{i}\right)$ is closed on $\mathbb{R}_{>0}^{3}$. Furthermore, for $u_{i}=\ln x_{i}$, the integral $F(u)=\int_{0}^{u} \sum_{i=1}^{3} a_{i} d u_{i}$ is a $C^{1}$-smooth convex function in $\left(u_{1}, u_{2}, u_{3}\right)$ on $\mathbb{R}^{3}$ so that $F$ is strictly convex when restricted to $\left\{u \in \mathbb{R}^{3} \mid u_{1}+u_{2}+u_{3}=\right.$ $\left.0, e^{u_{i}}+e^{u_{j}}>e^{u_{k}}\right\}$ and $F(u+(k, k, k))=F(u)+k \pi$ for all $k \in \mathbb{R}$.

For a generalized decorated tetrahedron of length vector $l=\left(l_{12}, \ldots, l_{34}\right) \in \mathbb{R}^{6}$, the dihedral angle $\alpha_{i j}$ at the edge $v_{i} v_{j}$ is defined to be the inner angle of the generalized Euclidean triangle of edge lengths $e^{\left(l_{i j}+l_{k h}\right) / 2}, e^{\left(l_{i k}+l_{j h}\right) / 2}$ and $e^{\left(l_{i h}+l_{j k}\right) / 2}$ so that $\alpha_{i j}$ is opposite to the edge of length $e^{\left(l_{i j}+l_{k h}\right) / 2}$ for $i, j, k, h$ distinct. Hence the dihedral angles $\alpha_{i j}=\alpha_{k h},\{i, j, k, h\}=\{1,2,3,4\}$, are continuous functions on $\mathbb{R}^{6}$.

We study the properties of volume functions and co-volume functions on generalized decorated ideal tetrahedra. Recall that the Lobachevsky function is defined as $\Lambda(x)=\int_{0}^{x} \ln |2 \sin t| d t$. The volume of a generalized decorated tetrahedron $\left\{v_{1}, v_{2}, v_{3}, v_{4}\right\}$ of lengths $l_{i j}$, denoted by $\operatorname{vol}(l)$, is defined to be $\frac{1}{2} \sum_{i<j} \Lambda\left(\alpha_{i j}(l)\right)$, where $\alpha_{i j}$ is the extended dihedral angle at edge $e_{i j}=v_{i} v_{j}$. By this definition, $\operatorname{vol}(l)$ is the hyperbolic volume of the underlying ideal tetrahedron if $l \in \mathscr{L} ; \operatorname{vol}(l)=0$, otherwise. The co-volume of a generalized decorated tetrahedron of lengths $l_{i j}$ is defined as

$$
\operatorname{cov}(l):=2 \operatorname{vol}(l)+\sum_{1 \leq i<j \leq 4} \alpha_{i j} l_{i j} .
$$

Proposition 2.3 ([[LY18] $)$. The co-volume function cov $: \mathbb{R}^{6} \rightarrow \mathbb{R}$ is a $C^{1}$ - smooth convex function so that for any $1 \leq i<j \leq 4$,

$$
\frac{\partial \operatorname{cov}(l)}{\partial l_{i j}}=\alpha_{i j} .
$$

For any $\alpha \in \mathbb{R}^{6}$ satisfying $\alpha_{i j}=\alpha_{k h}$ and $\alpha_{i j}+\alpha_{i k}+\alpha_{i h}=\pi$ for $\{i, j, k, l\}=\{1,2,3,4\}$, we define

$$
F_{\alpha}: \mathbb{R}^{6} \rightarrow \mathbb{R}, \quad l \mapsto F_{\alpha}(l)=\operatorname{cov}(l)-\alpha \cdot l .
$$

The group $\mathbb{R}^{4}$ acts on $\mathbb{R}^{6}$ as follows: for any $w \in \mathbb{R}^{4}, l \in \mathbb{R}^{6}$,

$$
(w+l)_{i j}:=l_{i j}+w_{i}+w_{j}, \quad \forall 1 \leq i<j \leq 4 .
$$

Then one can show that $F_{\alpha}$ is invariant under the action, i.e.

$$
F_{\alpha}(w+l)=F_{\alpha}(l), \quad \forall l \in \mathbb{R}^{6}, w \in \mathbb{R}^{4} .
$$


We denote by $\widehat{\mathbb{R}^{4}}:=\left\{w+0: w \in \mathbb{R}^{4}\right\}$ with $0 \in \mathbb{R}^{6}$, which is a 4-dimensional linear subspace of $\mathbb{R}^{6}$, and by $\mathbb{R}^{6} / \widehat{\mathbb{R}^{4}}$ the orthogonal subspace of $\widehat{\mathbb{R}^{4}}$ w.r.t. the standard inner product. We prove the following result.

Lemma 2.4. The co-volume function cov $: \mathbb{R}^{6} \rightarrow \mathbb{R}$ is smooth on $\mathscr{L}$. The restriction function cov $\left.\right|_{\mathbb{R}^{6} / \widehat{\mathbb{R}^{4}}}$ is strictly convex in $\mathscr{L} \cap \mathbb{R}^{6} / \widehat{\mathbb{R}^{4}}$. In fact, for any $l \in \mathscr{L}$, the kernel of Hess $\left.($ cov $)\right|_{l}$ is $\widehat{\mathbb{R}^{4}}$ and $\left.\operatorname{Hess}(\operatorname{cov})\right|_{l}$ is positive definite in $\mathbb{R}^{6} / \widehat{\mathbb{R}^{4}}$.

Proof. For $\alpha=\left(\frac{\pi}{3}, \frac{\pi}{3}, \cdots, \frac{\pi}{3}\right)$, consider the function $F_{\alpha}$ on $\mathbb{R}^{6}$. For any $l \in \mathbb{R}^{6}$, since $F_{\alpha}$ is invariant under the action of $\mathbb{R}^{4}, \widehat{\mathbb{R}}^{4}$ is contained in the kernel of $\left.\operatorname{Hess}\left(F_{\alpha}\right)\right|_{l}=\left.\operatorname{Hess}(\operatorname{cov})\right|_{l}$. By [Yan19, Theorem 2.14], the rank of Hess $\left.(c o v)\right|_{l}$ is two. Since cov is convex by Proposition 2.3. the kernel of Hess $\left.($ cov $)\right|_{l}$ is $\widehat{\mathbb{R}^{4}}$. This implies that Hess $\left.($ cov $)\right|_{l}$ is positive definite in $\mathbb{R}^{6} / \widehat{\mathbb{R}^{4}}$. The result follows.

Now we consider the properties of generalized decorated metric on pseudo 3-manifolds with triangulation. Let $(M, \mathcal{T})=\mathscr{T} / \sim$ be a closed pseudo 3 -manifold, where $\mathscr{T}$ be the disjoint union of tetrahedra $T_{1} \sqcup \cdots \sqcup T_{t}$. We denote by $T=T(\mathcal{T})$ the set of tetrahedra in $\mathscr{T}$. A quad in the triangulation $\mathcal{T}$ is a pair of opposite edges in a tetrahedron in $T$ and we write $\square=\square(\mathcal{T}$ ) for the set of all quads in $\mathcal{T}$. For $q \in \square, e \in E$ and $\sigma \in T$, we use $q \subset \sigma$ to denote that the quad $q$ is contained in the tetrahedron $\sigma$ and use $q \sim e$ or $e \sim q$ to denote that $e \subset q$. For any $\sigma \in T$ and any generalized decorated metric $l$, we denote by $l_{\sigma} \in \mathbb{R}^{6}$ the restriction of the metric $l$ on $\sigma$.

Definition 2.5. An angle assignment on $(M, \mathcal{T})$ is a map: $\alpha: \square \rightarrow \mathbb{R}_{\geq 0}$ so that for each tetrahedron $\sigma \in T, \sum_{q \subset \sigma} \alpha(q)=\pi$. The cone angle of $\alpha$ is defined to be $k_{\alpha}: E \rightarrow \mathbb{R}_{\geq 0}$ where

$$
k_{\alpha}(e)=\sum_{q \sim e} \alpha(q) \text {. }
$$

For a generalized decorated metric $l$ on $(M, \mathcal{T})$, it associates with an angle assignment $\alpha=\alpha_{l}$ : for any $q \in \square, \alpha(q)$ is defined as the extended dihedral angle at an edge e such that $e \sim q$ in the tetrahedron with generalized decorated metric induced by $l$. We write $k_{l}=k_{\alpha_{l}}$ for the cone angles at edges.

Using the above notation, the generalized Ricci curvature is given by

$$
\widetilde{K}_{e}(l)=2 \pi-k_{l}(e), \quad \forall e \in E .
$$

Definition 2.6. For a generalized decorated metric $l$ on $(M, \mathcal{T})$, the volume (resp. co-volume) function for the metric $l$ is defined as

$$
\operatorname{vol}(l)=\sum_{\sigma \in T} \operatorname{vol}_{\sigma}(l) \quad\left(\text { resp. } \operatorname{cov}(l)=\sum_{\sigma \in T} \operatorname{cov}_{\sigma}(l)\right),
$$

where $\operatorname{vol}_{\sigma}(l)=\operatorname{vol}\left(l_{\sigma}\right)$ (resp. $\operatorname{cov}_{\sigma}(l)=\operatorname{cov}\left(l_{\sigma}\right)$ ) is the volume (resp. co-volume) of the tetrahedron $\sigma$ endowed with the metric $l_{\sigma}$. 
Hence,

$$
\begin{gathered}
\operatorname{vol}(l)=\sum_{q \in \square} \Lambda\left(\alpha_{l}(q)\right), \\
\operatorname{cov}(l)=2 \operatorname{vol}(l)+l \cdot k_{l}=\sum_{\sigma \in T} \sum_{q \subset \sigma}\left[2 \Lambda\left(\alpha_{l}(q)\right)+\alpha_{l}(q) \sum_{e \sim q} l(e)\right] .
\end{gathered}
$$

The action of $\mathbb{R}^{V}$ on $\mathbb{R}^{E}$ is defined as follows: for any $w \in \mathbb{R}^{V}, l \in \mathbb{R}^{E}$,

$$
(w+l)_{e}:=l_{e}+w\left(e_{+}\right)+w\left(e_{-}\right), \quad \forall e \in E,
$$

where $e_{+}$and $e_{-}$are end-vertices of $e$. We denote by $\widehat{\mathbb{R}^{V}}:=\left\{w+0: w \in \mathbb{R}^{V}\right\}$ with $0 \in \mathbb{R}^{E}$, and by $\mathbb{R}^{E} / \widehat{\mathbb{R}^{V}}$ the orthogonal subspace of $\widehat{\mathbb{R}^{V}}$ w.r.t. the standard inner product. For any $l_{0} \in \mathbb{R}^{E}$, we set

$$
F_{l_{0}}(l):=\operatorname{cov}(l)-k_{l_{0}} \cdot l, \quad \forall l \in \mathbb{R}^{E} .
$$

Luo and Yang proved the following proposition.

Proposition 2.7 (Proposition 3.4 in [LY18]). (1) For any $l_{0} \in \mathbb{R}^{E}, F_{l_{0}}$ is invariant under the action of $\mathbb{R}^{V}$, i.e.

$$
F_{l_{0}}(w+l)=F_{l_{0}}(l), \quad \forall l \in \mathbb{R}^{E}, w \in \mathbb{R}^{V} .
$$

(2) For any $l_{0} \in \mathscr{L}(M, \mathcal{T})$,

$$
\lim _{l \in \mathbb{R}^{E} / \mathbb{R}^{V}, l \rightarrow \infty} F_{l_{0}}(l)=+\infty .
$$

It was proved by Luo and Yang [LY18] that the co-volume function cov $: \mathbb{R}^{E} \rightarrow \mathbb{R}$ is $C^{1}$ smooth and convex, which is smooth on $\mathscr{L}(M, \mathcal{T})$. We prove the following lemma.

Lemma 2.8. The restriction function cov $\left.\right|_{\mathbb{R}^{E} / \widehat{\mathbb{R}}^{V}}$ is strictly convex in $\mathscr{L}(M, \mathcal{T}) \cap \mathbb{R}^{E} / \widehat{\mathbb{R}^{V}}$.

Proof. Consider $F_{0}: \mathbb{R}^{E} \rightarrow \mathbb{R}$ where $0 \in \mathbb{R}^{E}$. For any $l \in \mathbb{R}^{E}$,

$$
\left.\operatorname{Hess}\left(F_{0}\right)\right|_{l}=\left.\operatorname{Hess}(\operatorname{cov})\right|_{l} \text {. }
$$

Since $F_{0}$ is invariant under the action of $\mathbb{R}^{V}, \widehat{\mathbb{R}^{V}}$ is contained in the kernel of Hess $\left.($ cov $)\right|_{l}$. Since cov is convex on $\mathbb{R}^{E}$, it suffices to prove that the kernel of $\left.H e s s(\operatorname{cov})\right|_{l}$ is exactly $\widehat{\mathbb{R} V}$, which implies that $\left.\operatorname{Hess}(\operatorname{cov})\right|_{l}$ is positive definite on $\mathbb{R}^{E} / \widehat{\mathbb{R}^{V}}$. As a consequence, cov $\left.\right|_{\mathbb{R}^{E} / \widehat{\mathbb{R}}^{V}}$ is strictly convex in $\mathscr{L}(M, \mathcal{T}) \cap \mathbb{R}^{E} / \widehat{\mathbb{R}^{V}}$.

Take any $x \in \mathbb{R}^{E}$ in the kernel of Hess $\left.($ cov $)\right|_{l}$. We need to show that $x \in \widehat{\mathbb{R}^{V}}$.

$$
\begin{aligned}
0 & =x^{T}\left(\left.\operatorname{Hess}(\operatorname{cov})\right|_{l}\right) x=x^{T}\left(\left.\sum_{\sigma \in T} \operatorname{Hess}\left(\operatorname{cov}_{\sigma}\right)\right|_{l}\right) x \\
& =\sum_{\sigma \in T} x_{\sigma}^{T}\left(\left.\operatorname{Hess}(\operatorname{cov})\right|_{l_{\sigma}}\right) x_{\sigma} \geq 0
\end{aligned}
$$


where $x_{\sigma}$ is the restriction of $x$ to $\sigma$. Hence $x_{\sigma}$ is in the kernel of Hess $\left.($ cov $)\right|_{l_{\sigma}}$. By Lemma 2.4, there exists $w_{\sigma} \in \mathbb{R}^{4}$, a function on vertices of $\sigma$, such that

$$
x_{\sigma}=w_{\sigma}+0, \quad 0 \in \mathbb{R}^{6} .
$$

By Neumann's Lemma [Neu92], see also [Cho04, p.1354] or [LY18, Lemma 3.3], $w_{\sigma}, \sigma \in T$, are consistent, i.e. for two tetrahedra $\sigma_{1}, \sigma_{2}$ sharing a vertex $v, w_{\sigma_{1}}(v)=w_{\sigma_{2}}(v)$. Hence, there exists a function $w \in \mathbb{R}^{V}$ such that the restriction of $w$ to $\sigma$ is $w_{\sigma}$. So that $x=w+0,0 \in \mathbb{R}^{V}$ and $x \in \widehat{\mathbb{R}^{V}}$. This proves the result.

\section{THE COMBINATORIAL RICCI FLOW}

Let $(M, \mathcal{T})=\mathscr{T} / \sim$ be a closed pseudo 3-manifold. Let $E=E(\mathcal{T})=\left\{e_{1}, e_{2}, \cdots, e_{m}\right\}$, where $m$ is the number of edges. To simplify the notation, we write $E=\{1,2, \cdots, m\}$, that is, each edge $e_{i}$ is replaced by the index $i$. In this way, each sub-index of an edge $e_{i}$ is replaced by $i$. For example, we write the edge length vector $l=\left(l_{1}, l_{2}, \cdots l_{m}\right)$ and the Ricci curvature $K(l)=\left(K_{1}(l), K_{2}(l), \cdots, K_{m}(l)\right)$, etc.

The combinatorial Ricci flow (1.1) in $\mathscr{L}(M, \mathcal{T})$ can be written as follows:

$$
\left\{\begin{array}{l}
\frac{d l_{i}(t)}{d t}=K_{i}(l(t)), \quad \forall i \in E, t \geq 0, \\
l(0)=l_{0}
\end{array}\right.
$$

where $l_{0} \in \mathscr{L}(M, \mathcal{T})$ and $l(t) \in \mathscr{L}(M, \mathcal{T}), \forall t>0$. Since $\mathscr{L}(M, \mathcal{T})$ is an open subset in $\mathbb{R}^{E}$ and $K(l)$ is smooth on $\mathscr{L}(M, \mathcal{T})$, Picard's theorem in ordinary differential equations yields the following.

Theorem 3.1. For a closed pseudo 3-manifold $(M, \mathcal{T})$, for any initial data $l_{0} \in \mathscr{L}(M, \mathcal{T})$, the solution $\{l(t) \mid t \in[0, T)\} \subset \mathscr{L}(M, \mathcal{T})$ to the combinatorial Ricci flow (1.1) exists and is unique on the maximal existence interval $[0, T)$ with $0<T \leq \infty$.

We define the functional

$$
H: \mathscr{L}(M, \mathcal{T}) \rightarrow \mathbb{R}, \quad l \mapsto H(l)=\operatorname{cov}(l)-2 \pi \sum_{i=1}^{m} l_{i}
$$

By Proposition 2.3.

$$
\begin{aligned}
\frac{\partial H}{\partial l_{j}} & =\frac{\partial \operatorname{cov}(l)}{\partial l_{j}}-2 \pi=\sum_{\sigma \in T} \frac{\partial \operatorname{cov}_{\sigma}(l)}{\partial l_{j}}-2 \pi \\
& =\sum_{q \sim j, q \in \square} \alpha(q)-2 \pi \\
& =-K_{j}
\end{aligned}
$$


By this result, $l \in \mathscr{L}(M, \mathcal{T})$ has zero Ricci curvature if and only if $l$ is a critical point of the functional $H$.

Hence, the combinatorial Ricci flow in (1.1) can be written as

$$
\left\{\begin{array}{l}
\frac{\partial l_{i}(t)}{\partial t}=-\partial_{i} H(l), \\
l(0)=l_{0} .
\end{array}\right.
$$

This implies that the combinatorial Ricci flow is the negative gradient flow of the functional $H$.

Proposition 3.2. The functional $H$ is non-increasing along the combinatorial Ricci flow (1.1), i.e. for any solution $l(t)$ to the flow (1.1),

$$
\frac{\partial H(l(t))}{\partial t} \leq 0
$$

Proof. By direct calculation,

$$
\frac{\partial H(l(t))}{\partial t}=-|K(l(t))|^{2} \leq 0
$$

Proposition 3.3. Let $l(t)$ be a solution to the combinatorial Ricci flow (1.1) which converges to $\bar{l} \in \mathscr{L}$. Then $K(\bar{l})=0$.

Proof. This is well-known in classical ODE theory. For the convenience of readers, we include the proof here. By Proposition 3.2, $H(l(t))$ is non-increasing. Moreover, $\{H(l(t)): t \geq 0\}$ is bounded, since $H$ is continuous on $\mathscr{L}$ and $l(t) \rightarrow \bar{l}, t \rightarrow \infty$. Hence the following limit exists and is finite,

$$
\lim _{t \rightarrow \infty} H(l(t))=C .
$$

Consider the sequence $\{H(l(n))\}_{n=1}^{\infty}$. By the mean value theorem, for any $n \geq 1$ there exists $t_{n} \in(n, n+1)$ such that

$$
H(l(n+1))-H(l(n))=\left.\frac{d}{d t}\right|_{t=t_{n}} H(l(t))=-\left|K\left(l\left(t_{n}\right)\right)\right|^{2} .
$$

Note that $\lim _{n \rightarrow \infty} H(l(n+1))-H(l(n))=0$. We get

$$
\lim _{n \rightarrow \infty} K_{i}\left(l\left(t_{n}\right)\right)=0, \quad \forall i \in E .
$$

Since $l\left(t_{n}\right) \rightarrow \bar{l}$ as $n \rightarrow \infty$, the continuity of $K_{i}$ yields that $K_{i}(\bar{l})=0$ for any $i \in E$. This proves the proposition.

Next we consider the extended Ricci flow on the set of generalized decorated metrics, i.e. $\mathbb{R}^{E}$. The extended Ricci flow (1.2) can be written as

$$
\left\{\begin{array}{l}
\frac{\partial l_{i}(t)}{\partial t}=\widetilde{K}_{i}(l(t)) \\
l(0)=l_{0}
\end{array}\right.
$$


where $l_{0} \in \mathbb{R}^{E}$ and $l(t) \in \mathbb{R}^{E}, \forall t>0$.

We define the extended functional $\widetilde{H}$ on $\mathbb{R}^{E}$ by

$$
\widetilde{H}(l)=\operatorname{cov}(l)-2 \pi \sum_{i=1}^{m} l_{i} .
$$

Note that $\widetilde{H}$ is a $C^{1}$ - smooth convex functional on $\mathbb{R}^{E}$ and it extends the functional $H$ on $\mathscr{L}(M, \mathcal{T})$. Direct calculation yields that

$$
\frac{\partial \widetilde{H}}{\partial l_{i}}=-\widetilde{K}_{i}, \quad \forall i \in E .
$$

Hence, $l \in \mathbb{R}^{E}$ has zero generalized Ricci curvature if and only if it is a critical point of the functional $\widetilde{H}$. As in Proposition 3.2, one can show that the functional $\widetilde{H}$ is non-increasing along the extended Ricci flow (1.2).

Note that if a closed pseudo 3-manifold $(M, \mathcal{T})$ supports a zero-curvature generalized decorated metric $\hat{l}$, then

$$
\widetilde{H}(l)=F_{\hat{l}}(l),
$$

where $F_{\hat{l}}$ is defined in (2.3). In particular, $\widetilde{H}$ is invariant under the action of $\mathbb{R}^{V}$ on $\mathbb{R}^{E}$ by Proposition 2.7.

Proposition 3.4. Let $(M, \mathcal{T})$ be a closed pseudo 3-manifold which has a zero-curvature generalized decorated metric. Then the solution of the extended Ricci flow is compatible with the action $\mathbb{R}^{V}$ on $\mathbb{R}^{E}$, i.e., for any initial data $l_{0} \in \mathbb{R}^{E}$ with $l_{0}=w+l_{0}^{\top}$, where $w \in \mathbb{R}^{V}$ and $l_{0}^{\top}$ is the projection of $l_{0}$ to $\mathbb{R}^{E} / \widehat{\mathbb{R}^{V}}$, then the solution $l(t)$ of the extended Ricci flow with the initial data $l_{0}$ satisfies

$$
l(t)=w+l^{\top}(t), \forall t \in[0, \infty),
$$

where $l^{\top}(t)$ is the solution of the extended Ricci flow with the initial data $l_{0}^{\top}$. Moreover, $l^{\top}(t) \in$ $\mathbb{R}^{E} / \widehat{\mathbb{R}^{V}}$ for all $t \in[0, \infty)$.

Proof. Note that $\widetilde{H}$ is invariant under the action of $\mathbb{R}^{V}$ on $\mathbb{R}^{E}$ and the extended Ricci flow is the negative gradient flow of $\widetilde{H}$. By these observation, for any initial data $l_{0}^{\top} \in \mathbb{R}^{E} / \widehat{\mathbb{R}^{V}}$, there is a solution of the extended Ricci flow $l^{\top}(t) \in \mathbb{R}^{E} / \widehat{\mathbb{R}^{V}}$ for all $t \in[0, \infty)$. In fact, it is induced by the negative gradient flow of $\left.\widetilde{H}\right|_{\mathbb{R}^{E} / \mathbb{R}^{V}}$ on $\mathbb{R}^{E} / \widehat{\mathbb{R}^{V}}$. By the uniqueness of the solution of the extended Ricci flow, we prove the above results.

Theorem 3.5 ([[LY18]). Let $(M, \mathcal{T})$ be a closed pseudo 3-manifold which has a zero-curvature decorated metric. Then

$$
\lim _{l \in \mathbb{R}^{E} / \mathbb{R}^{V}, l \rightarrow \infty} \tilde{H}(l)=+\infty .
$$

In particular, $\widetilde{H}(l)$ is proper on $\mathbb{R}^{E} / \widehat{\mathbb{R}^{V}}$. 
Proof. By $\widetilde{H}(l)=F_{l_{0}}(l)$, where $K\left(l_{0}\right)=0$, the results follow from Proposition 2.7 .

The following rigidity result was proved by Luo and Yang.

Theorem 3.6 (Theorem 1.2 in [LY18]). Let $(M, \mathcal{T})$ be a closed pseudo 3-manifold which has a zero-curvature decorated metric $l_{0} \in \mathscr{L}(M, \mathcal{T})$. Then the set of zero-curvature generalized decorated metrics on $(M, \mathcal{T})$ is unique up to the action $\mathbb{R}^{V}$ on $\mathbb{R}^{E}$, i.e.

$$
\left\{l \in \mathbb{R}^{E}: \widetilde{K}(l)=0\right\}=\left\{w+l_{0}: w \in \mathbb{R}^{V}\right\} .
$$

Proof. Here we give an alternative proof using the strict convexity of the functional $\widetilde{H}$. Note that $\widetilde{H}$ is invariant under the action of $\mathbb{R}^{V}$ on $\mathbb{R}^{E}$. If $l \in \mathbb{R}^{E}$ has zero Ricci curvature, then so does $w+l$ for any $w \in \mathbb{R}^{V}$. Let $l_{0}^{\top}$ be the projection of $l_{0} \in \mathscr{L}(M, \mathcal{T})$ to $\mathbb{R}^{E} / \widehat{\mathbb{R}^{V}}$. Then $l_{0}^{\top} \in \mathscr{L}(M, \mathcal{T})$ and it also has zero Ricci curvature. Note that zero-curvature generalized decorated metrics are critical points of the functional $\widetilde{H}$. Since $\widetilde{H}$ is convex, all of them are in fact minimizers. By Lemma 2.8, $\widetilde{H}$ is strictly convex in $\mathscr{L}(M, \mathcal{T}) \cap \mathbb{R}^{E} / \widehat{\mathbb{R}^{V}}$. Since $l_{0}^{\top} \in \mathscr{L}(M, \mathcal{T}) \cap \mathbb{R}^{E} / \widehat{\mathbb{R}^{V}}$ is a minimizer of $\widetilde{H}$, it is the unique critical point of $\widetilde{H}$ on $\mathbb{R}^{E} / \widehat{\mathbb{R}^{V}}$. This proves the result.

Next, we prove the long-time existence and uniqueness of the extended Ricci flow.

Proof of Theorem 1.3 Since the extended Ricci curvature $\widetilde{K}(l)$ is continuous on $\mathbb{R}^{E}$, by Peano's existence theorem in classical ODE theory, the extended Ricci flow (1.2) has at least one solution on some interval $[0, T)$, with the maximal existence time $T$. Furthermore, $|\widetilde{K}(l)|$ are uniformly bounded by a constant $C>0$ which depends on the triangulation $\mathcal{T}$. Hence

$$
|l(t)| \leq|l(0)|+C t
$$

for all $t \geq 0$, which implies that $T=\infty$.

Next, we prove the uniqueness of the solution of the extended Ricci flow. Let $l(t)$ and $\hat{l}(t)$ be two solutions of of the extended Ricci flow with initial data $l_{0}$. We need to prove that $l(t)=\hat{l}(t)$ for any $t \in[0, \infty)$. Set

$$
h(t)=|l(t)-\hat{l}(t)|^{2} \geq 0, \quad t \in[0, \infty) .
$$

Then,

$$
\begin{aligned}
h^{\prime}(t) & =2(l(t)-\hat{l}(t)) \cdot(\widetilde{K}(l(t))-\widetilde{K}(\hat{l}(t))) \\
& =-2(l(t)-\hat{l}(t)) \cdot(\nabla \widetilde{H}(l(t))-\nabla \widetilde{H}(\hat{l}(t))) \\
& \leq 0,
\end{aligned}
$$

where the last inequality follows from the convexity of a $C^{1}$ functional $\widetilde{H}$. For $h(0)=0, h(t)=$ 0 , i.e. $l(t)=\hat{l}(t)$, for any $t \in[0, \infty)$. This proves the result.

By the same argument as in Proposition 3.3, one can prove the following. 
Proposition 3.7. If a solution $l(t)$ of the extended Ricci flow (1.2) converges to some $\widetilde{l} \in \mathbb{R}^{E}$ as $t \rightarrow+\infty$. Then $\widetilde{K}(\widetilde{l})=0$.

We are ready to prove the main result.

Proof of Theorem 1.4 We prove the first assertion. Suppose that it is not true, then there exists a constant $C$ such that $|l(t)| \leq C, t \in[0, \infty)$.

Let $\phi(t)=\widetilde{H}(l(t))$. Then $\phi(t)$ is bounded on $t \in[0, \infty)$. Since $\phi(t)$ is non-increasing, the following limit exists

$$
\lim _{t \rightarrow \infty} \phi(t)=C \in \mathbb{R} .
$$

By the mean value theorem, there are $\xi_{n} \in(n, n+1)$ such that

$$
\phi^{\prime}\left(\xi_{n}\right)=\phi(n+1)-\phi(n) \rightarrow 0, \quad n \rightarrow \infty
$$

Since $\phi^{\prime}(t)=-|\widetilde{K}(l(t))|^{2}$,

$$
\widetilde{K}\left(l\left(\xi_{n}\right)\right) \rightarrow 0, \quad n \rightarrow \infty .
$$

By passing to a subsequence, $l\left(\xi_{n}\right) \rightarrow l_{\infty} \in \mathbb{R}^{E}$. This implies that

$$
\widetilde{K}\left(l_{\infty}\right)=0 .
$$

This yields a contradiction and proves the first assertion.

Now we prove the second assertion. By Proposition 3.3, we only need to prove that if there is a zero-curvature decorated metric, then $l(t)$ converges to a zero-curvature decorated metric exponentially fast. By Proposition 3.4, it suffices to prove the result for the initial data $l_{0} \in$ $\mathbb{R}^{E} / \widehat{\mathbb{R}^{V}}$. Hence the solution $l(t)$ of the extended Ricci flow satisfies $l(t) \in \mathbb{R}^{E} / \widehat{\mathbb{R}^{V}}$ for all $t \in$ $[0, \infty)$.

By Luo and Yang's rigidity theorem, Theorem 3.6, there is a unique zero-curvature decorated metric $\hat{l}$ in $\mathbb{R}^{E} / \widehat{\mathbb{R}^{V}}$. By Theorem 3.5 , the functional $\left.\widetilde{H}\right|_{\mathbb{R}^{E} / \widehat{\mathbb{R}^{V}}}$ is proper on $\mathbb{R}^{E} / \widehat{\mathbb{R}^{V}}$ and $\widetilde{H}$ is bounded from below.

For $\phi(t)=\widetilde{H}(l(t))$, since $\phi(t)$ is non-increasing, the following limit exists

$$
\lim _{t \rightarrow \infty} \phi(t)=C \in \mathbb{R} .
$$

By the properness of $\left.\widetilde{H}\right|_{\mathbb{R}^{E} / \widehat{\mathbb{R}^{V}}},\{l(t): t \in[0, \infty)\}$ is contained in a compact subset of $\mathbb{R}^{E} / \widehat{\mathbb{R}^{V}}$. By the mean value theorem, there is a sequence $\xi_{n} \in(n, n+1)$ such that

$$
-\left|\widetilde{K}\left(l\left(\xi_{n}\right)\right)\right|^{2}=\phi^{\prime}\left(\xi_{n}\right)=\phi(n+1)-\phi(n) \rightarrow 0, \quad n \rightarrow \infty .
$$

Passing to a subsequence, still denoted by $\xi_{n}$,

$$
l\left(\xi_{n}\right) \rightarrow l_{\infty}, \quad n \rightarrow \infty .
$$

By the continuity of $\widetilde{K}, \widetilde{K}\left(l_{\infty}\right)=0$. By Theorem 3.6 , we have

$$
l_{\infty}=\hat{l} \text {. }
$$


Hence for any neighbourhood $U$ of $\hat{l}$ in $\mathbb{R}^{E} / \widehat{\mathbb{R}^{V}}$, for sufficiently large $\xi_{n}$,

$$
l\left(\xi_{n}\right) \in U .
$$

By Lemma 2.8, $\operatorname{Hess}\left(\left.\widetilde{H}\right|_{\mathbb{R}^{E} / \mathbb{R}^{V}}\right)_{\hat{l}}$ is positive definite, which implies the critical point $\hat{l}$ is a local attractor of the extended Ricci flow restricted on $\mathbb{R}^{E} / \widehat{\mathbb{R}^{V}}$. By Lyapunov's theorem in the ODE theory, the flow $l(t)$ converges to $\hat{l}$ exponentially fast for any initial data. This proves the result.

Now we prove Theorem 1.6 .

Proof of Theorem 1.6. This follows from Theorem 1.4. The other statements are well-known results in hyperbolic geometry.

In the following we prove Corollary 1.7

Proof of Corollary 1.7 We claim that if there exists a zero-curvature generalized decorated metric $l$, then there exists a zero-curvature decorated metric $\hat{l}$ with constant length, i.e. $\hat{l}_{i}=\hat{l}_{j}$ for any $i, j \in E$. Since $(M, \mathcal{T})$ is edge-transitive and $l=\left(l_{1}, \cdots, l_{m}\right)$ has zero Ricci curvature, for any permutation $\eta \in S_{m}, l_{\eta}:=\left(l_{\eta(1)}, \cdots, l_{\eta(m)}\right)$ has zero Ricci curvature. Since the set of zerocurvature generalized decorated metrics $K$ consists of critical points of the convex functional $\widetilde{H}$, $K$ is a convex set in $\mathbb{R}^{E}$. Since $\hat{l}:=\left(\frac{1}{m} \sum_{i} l_{i}, \cdots, \frac{1}{m} \sum_{i} l_{i}\right)$ is in the convex hull of $\left\{l_{\eta}\right\}_{\eta \in S_{m}}$, $\hat{l} \in K$. This proves the claim.

For the assertion (1), $d_{i}=d \neq 6$ for any $i \in E$. We argue by contradiction. Suppose that there is a zero-curvature generalized decorated metric $l$, then by the claim there exists a zero-curvature decorated metric $\hat{l}$ with constant length. Hence for each edge $e$ and quad $q$ with $e \sim q, \alpha(q)=\frac{\pi}{3}$. This implies that

$$
K_{e}(\hat{l})=2 \pi-d \frac{\pi}{3} \neq 0 .
$$

This is a contradiction. Hence there exists no zero-curvature generalized decorated metrics, and the extended Ricci flow $l(t)$ diverges to infinity in subsequence by Theorem 1.4 .

For the assertion (2), $d_{i}=6$ for any $i \in E$. One can verify that $l=(1, \cdots, 1) \in \mathscr{L}(M, \mathcal{T})$ is a zero-curvature decorated metric. Then result follows from Theorem 1.4.

Next, we consider the prescribed Ricci curvature problem. The curvature map is defined as

$$
K: \mathscr{L}(M, \mathcal{T}) \rightarrow \mathbb{R}^{E}, l \mapsto K(l) .
$$

General questions are as follows: For $\bar{K} \in \mathbb{R}^{E}$, is there any $l \in \mathscr{L}(M, \mathcal{T})$ such that $K(l)=\bar{K}$ ? If there are some, how could one find the set $K^{-1}(\bar{K})$ ? The structure of $K^{-1}(\bar{K})$ is determined 
by Luo and Yang's rigidity theorem, Theorem 3.6. Namely, if there is $l_{0} \in \mathscr{L}(M, \mathcal{T})$ such that $K\left(l_{0}\right)=\bar{K}$, then

$$
K^{-1}(\bar{K}):=\left\{w+l_{0}: w \in \mathbb{R}^{V}\right\} .
$$

For the case $\bar{K}=0$, it reduces to the problem of zero Ricci curvature as before. To study the general problem, we introduce the following extended Ricci flow $l(t) \in \mathbb{R}^{E}$

$$
\frac{d}{d t} l(t)=\widetilde{K}(l(t))-\bar{K}, \quad \forall t \geq 0 .
$$

Note that if there is $l_{0} \in \mathscr{L}(M, \mathcal{T})$ of zero Ricci curvature, then it is a negative gradient flow of the convex functional $F_{l_{0}}$. Similar results as Theorem 1.3 and Theorem 1.4 can be derived accordingly. We omit the proofs here.

Define the discrete Laplace operator $\Delta=-\frac{\partial K}{\partial l}$. One may also consider the following combinatorial Calabi flow

$$
\frac{d l}{d t}=\Delta K
$$

which is the negative gradient flow of the combinatorial Calabi energy $C=\|K\|^{2} / 2$. Using similar methods as in [Ge12, Ge18], one can study the convergence of the flow and the existence of zero-curvature decorated metrics.

At the end, to realize our program about the hyperbolization of 3-manifolds by combinatorial Ricci flow methods, we need further to prove that suitable topological conditions (such as incompressible, atoroidal) are equivalent to the convergence of the extended combinatorial Ricci flow.

\section{REFERENCES}

[BPS15] Alexander I. Bobenko, Ulrich Pinkall, and Boris A. Springborn. Discrete conformal maps and ideal hyperbolic polyhedra. Geom. Topol., 19(4):2155-2215, 2015.

[Cho04] Young-Eun Choi. Positively oriented ideal triangulations on hyperbolic three-manifolds. Topology, 43(6):1345-1371, 2004.

[CKP01] Henry Cohn, Richard Kenyon, and James Propp. A variational principle for domino tilings. J. Amer. Math. Soc., 14(2):297-346, 2001.

[CL03] Bennett Chow and Feng Luo. Combinatorial Ricci flows on surfaces. J. Differential Geom., 63(1):97-129, 2003.

[CR96] Daryl Cooper and Igor Rivin. Combinatorial scalar curvature and rigidity of ball packings. Math. Res. Lett., 3(1):51-60, 1996.

[FGH20] Ke Feng, Huabin Ge, and Bobo Hua. Convergence of curvature flows for hyper-ideal polyhedral metrics. arXiv:2009.03731, 2020.

[Ge12] Huabin Ge. Combinatorial methods and geometric equations. PhD Thesis, Peking University, 2012.

[Ge18] Huabin Ge. Combinatorial Calabi flows on surfaces. Trans. Amer. Math. Soc., 370(2):1377-1391, 2018.

[GH20] Huabin Ge and Bobo Hua. 3-dimensional combinatorial Yamabe flow in hyperbolic background geometry. Trans. Amer. Math. Soc., 373(7):5111-5140, 2020.

[GJS18] Huabin Ge, Wenshuai Jiang, and Liangming Shen. On the deformation of ball packings. arXiv:1805.10573., 2018. 
[Gli05a] David Glickenstein. A combinatorial Yamabe flow in three dimensions. Topology, 44(4):791-808, 2005.

[Gli05b] David Glickenstein. A maximum principle for combinatorial Yamabe flow. Topology, 44(4):809-825, 2005.

[Luo05] Feng Luo. A combinatorial curvature flow for compact 3-manifolds with boundary. Electron. Res. Announc. Amer. Math. Soc., 11:12-20, 2005.

[Luo11a] Feng Luo. A note on complete hyperbolic structures on ideal triangulated 3-manifolds. In Topology and geometry in dimension three, volume 560 of Contemp. Math., pages 19-26. Amer. Math. Soc., Providence, RI, 2011.

[Luo11b] Feng Luo. Rigidity of polyhedral surfaces, III. Geom. Topol., 15(4):2299-2319, 2011.

[LY18] Feng Luo and Tian Yang. Volume and rigidity of hyperbolic polyhedral 3-manifolds. J. Topol., 11(1):129, 2018.

[Moi52] Edwin E. Moise. Affine structures in 3-manifolds. V. The triangulation theorem and Hauptvermutung. Ann. of Math. (2), 56:96-114, 1952.

[Neu92] Walter D. Neumann. Combinatorics of triangulations and the Chern-Simons invariant for hyperbolic 3manifolds. In Topology '90 (Columbus, OH, 1990), volume 1 of Ohio State Univ. Math. Res. Inst. Publ., pages 243-271. de Gruyter, Berlin, 1992.

[Pen87] R. C. Penner. The decorated Teichmüller space of punctured surfaces. Comm. Math. Phys., 113(2):299339, 1987.

[Thu79] William P. Thurston. Geometry and topology of 3-manifolds, volume 42. Lecture Notes, Princeton University, http://www.msri.org/publications/books/gt3m/, 1979.

[Yan19] Tianyu Yang. A combinatorial curvature flow for ideal triangulations. PhD thesis, The University of Melbourne, https://minerva-access.unimelb.edu.au/handle/11343/222445, 2019.

Ke Feng: School of Mathematical Sciences, University of Electronic Science and Technology of China; No.2006, Xiyuan Ave, West Hi-Tech Zone, Chengdu, Sichuan, 611731, P.R.China

E-mail address: youyouguzhe@126.com

Huabin Ge: School of Mathematics, Renmin University of China, Beijing, 100872, P.R. China

E-mail address: hbge@ruc.edu.cn

Bobo Hua: School of Mathematical Sciences, LMnS, Fudan University, Shanghai 200433, China; Shanghai Center for Mathematical Sciences, Fudan University, Shanghai 200433, China.

E-mail address: bobohua@ fudan.edu.cn 